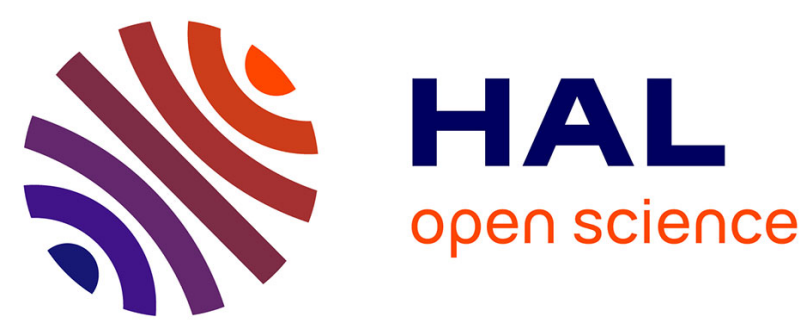

\title{
Characterisation of soils with stony inclusions using geoelectrical measurements
}

Etienne Rey, Denis Jongmans, Philippe, Gotteland, Stéphane Garambois

\section{To cite this version:}

Etienne Rey, Denis Jongmans, Philippe, Gotteland,, Stéphane Garambois. Characterisation of soils with stony inclusions using geoelectrical measurements. Journal of Applied Geophysics, 2006, 58, pp.188-201. 10.1016/j.jappgeo.2005.06.003 . insu-00196672

\section{HAL Id: insu-00196672 \\ https://hal-insu.archives-ouvertes.fr/insu-00196672}

Submitted on 13 Dec 2007

HAL is a multi-disciplinary open access archive for the deposit and dissemination of scientific research documents, whether they are published or not. The documents may come from teaching and research institutions in France or abroad, or from public or private research centers.
L'archive ouverte pluridisciplinaire $\mathbf{H A L}$, est destinée au dépôt et à la diffusion de documents scientifiques de niveau recherche, publiés ou non, émanant des établissements d'enseignement et de recherche français ou étrangers, des laboratoires publics ou privés. 


\title{
CHARACTERISATION OF SOILS
}

\section{WITH STONY INCLUSIONS USING GEOELECTRICAL \\ MEASUREMENTS}

\author{
Etienne Rey, Denis Jongmans*, \\ Philippe Gotteland, Stephane Garambois
}

L.I.R.I.G.M., Grenoble University,

Maison des Géosciences, BP 53X, 38041 Grenoble Cedex 9, France

* Corresponding author :

Tel. :+0033-4 76828117

Fax : +0033-4 76828070 .

E-mail adress : denis.jongmans@ujf-grenoble.fr 


\begin{abstract}
Characterisation and sampling of coarse heterogeneous soils is often impossible using common geotechnical in-situ tests once the soil contains particles with a diameter larger than a few decimetres. In this situation geophysical techniques - and particularly electrical measurements - can act as an alternative method for obtaining information about the ground characteristics. This paper deals with the use of electrical tomography on heterogeneous diphasic media consisting of resistive inclusions embedded in a conductive matrix. The adopted approach articulates in three steps: numerical modelling, measurements on a small-scale physical model, and field measurements. Electrical measurements were simulated using finite element analyses, on a numerical model containing a random concentration of inclusions varying from 0 to $40 \%$. It is shown that for electrode spacing 8 times greater than the radius of inclusions, the equivalent homogeneous resistivity is obtained. In this condition, average measured resistivity is a function of the concentration of inclusions, in agreement with the theoretical laws. To apply these results on real data, a small-scale physical model has been built, where electrical measurements were conducted both on the model and on each phase. From these laboratory measurements, a very satisfying estimation of the percentage of inclusions has been obtained. Finally, the methodology applied to a real experimental site composed of alluvial fan deposits made of limestone rocks embedded in a clayey matrix. The estimated percentage of rock particles obtained via electrical measurements was in accordance with the real grain size distribution.
\end{abstract}




\section{INTRODUCTION}

Coarse soils such as scree-slopes, till, alluvial fans, debris flows or dope deposits are commonly present in mountainous areas. The presence of decimetre to meter size pebbles or rocks in these deposits makes them difficult to characterise using traditional geotechnical techniques. First, in-situ tests like penetration tests or boreholes are hard to perform in such loose and heterogeneous formations. Second, the presence of decimetric rocks would require an elementary representative volume of soil (e.g. Standard NFP 94-056) much too large (several tens of cubic meters) for the traditional soil test devices. These materials are generally characterised by a two-phases procedure which consists in: (i) removing the grains larger than 100 millimetres in size and (ii) performing geotechnical tests on the remaining soil. However, such a procedure results in an underestimate of the mechanical characteristics of the geological formation. Indeed the angle of friction increases with the maximum particle size (Fagnoul \& Bonnechere, 1969; Bourdeau, 1997) and with the proportion of grains, due to interlocking (Holtz, 1961). As classical geotechnical techniques are not efficient to characterize coarse materials at a scale which is consistent with their maximum particle size, alternative methods have to be developed, at least to estimate the overall proportion of grains, which constitute an interesting information in terms of mechanical properties.

Geophysical methods, which are non-intrusive and able to investigate a large volume of soil, may constitute an interesting alternative to in-situ geotechnical tests. Quick and relatively cheap to perform, they are able to characterise coarse formations as a whole and to detect lateral variations of geophysical properties (P-wave velocity, S-wave velocity, electrical resistivity...). Their main drawbacks are the decrease of the resolution with depth and that mechanical properties of the soil 
are not directly accessed. However, empirical correlations between geophysical and geotechnical parameters have been proposed, particularly between shear wave velocity and strength properties (e.g., Mayne \& Rix, 1995).

One of the main geophysical methods for deriving shear wave velocity profiles is the inversion of surface waves (Jongmans \& Demanet, 1993). Several attempts were made to apply this method when characterising heterogeneous materials or "hard-to-sample" soils (Stockoe et al., 1988). Recently, Chammas et al. (2003) showed that Rayleigh waves are well-suited to the determination of shear wave velocity of a heterogeneous soil layer. Indeed, for wavelengths 7.5 times greater than the radius of circular inclusions, and within a concentration range from 0 to $50 \%$, surface waves homogenize the soil in accordance with the multiple-scattering homogenization theory (Christensen \& Lo, 1979). By simulating via finite-element analysis, several random distributions of inclusions made of limestone or sandstone in a sandy matrix, Chammas et al. (2003) evinced the increase of the shear wave velocity Vs with the concentration of stiff inclusions (for velocity contrasts ranging from 2.4 to 5.5). This relationship, validated using a small-scale model (Abraham et al., 2004) at two different concentrations (19\% and 35\%), allows one to estimate the concentration of inclusions present in a soil using only seismic measurement techniques.

Electrical resistivity (or conductivity), which is an easy geophysical parameter to measure in the field, exhibits a wide range of values in nature, from $1 \Omega \mathrm{m}$ in clay or contaminated soil to more than $10^{4}$ $\Omega \mathrm{m}$ in rocks like limestone or granite (Reynolds, 1997). Even if electrical and mechanical properties of the soils are not directly linked, electrical resistivity of a material is sensitive to the presence of resistive or conductive inclusions. For the interpretation of borehole resistivity logs in porous rocks, 
many authors studied the link between the effective resistivity (macroscopic, with regard to the size of heterogeneities) of the saturated porous rocks and its characteristics (porosity, fluid conductivity...). Many decades ago, Archie (1942) performed DC electrical measurements on brine-saturated cores for a wide variety of sand formations and proposed a simple empirical relationship (equation 1) between the effective (equivalent homogeneous) resistivity $\rho_{0}$ of the rock, the resistivity of the fluid $\rho_{f}$, the cementation factor $m$ and the porosity $\phi$.

$$
F=\frac{\rho_{0}}{\rho_{f}}=\phi^{-m}
$$

The ratio $\mathrm{F}$ is called the formation factor. According to Archie, $m$ varies from 1.3 for unconsolidated sands to 1.8 to 2 for consolidated sandstones. From their electrical measurements on unconsolidated marine sands obeying to Archie's law, Jackson et al. (1978) concluded that exponent $m$ is entirely dependent upon the shape of the resistive particles, varying from 1.2 for spheres to 1.9 for platey shell fragments. A possible explanation for these variations is that the $m$ exponent value increases when the conduction paths are more tortuous (Jackson et al., 1978). By performing measurements on artificial porous rocks obtained by the fusion of glass beads, Sen et al (1981) attributed a value of 1.5 to the cementation factor in accordance with their homogenisation model. Geometrical considerations conducted Mendelson and Cohen (1982) to demonstrate that $m$ takes a value of 1.5 for spherical rock particles spread in three dimensions, in accordance with the results of Sen et al. (1981). Cementation factor $m$ takes on a value of 2 in the case of cylinders with their axes perpendicular to electrical field (Sen et al., 1981) and for circular grains in two dimensions (Mendelson \& Cohen, 1982). In these Differential Effective Medium approaches, the desired concentration of inclusions is reached by adding infinitesimal increments of dispersed inclusions to a 
host conductive continuous material. However the main limit of these models lies in the assumption that the inclusions are infinetely resistive. Based on the equation of Hanaï-Bruggeman, Bussian (1983) proposed an expression (equation 2) of the effective resistivity $\rho_{0}$ for a diphasic medium composed of resistive inclusions $\left(\rho_{i}\right)$ embedded in a conductive matrix $\left(\rho_{f}\right)$.

$$
\frac{\rho_{0}}{\rho_{f}}=\phi^{-m}\left(\frac{1-\frac{\rho_{f}}{\rho_{i}}}{1-\frac{\rho_{0}}{\rho_{i}}}\right)^{-m}
$$

where $\phi$ is now the fractional volume of continuous medium. Equation (2) can be rewritten as equation (3), which provides a direct estimation of the composition of a diphasic medium through its effective $\left(\rho_{0}\right)$ and individual $\left(\rho_{i}, \rho_{f}\right)$ electrical properties

$$
\phi=\frac{\left(\frac{\rho_{0}}{\rho_{f}}\right)^{-1 / m}-\left(\frac{\rho_{f}}{\rho_{i}}\right)\left(\frac{\rho_{0}}{\rho_{f}}\right)^{(m-1) / m}}{1-\left(\frac{\rho_{f}}{\rho_{i}}\right)}
$$

Alluvial fan deposits found in the Grenoble area (France) are mainly made of a mixing of calcareous pebbles and clay (figure 1a), and can be considered as diphasic media composed of resistive inclusions embedded in a conductive matrix (figure 1b). Under this assumption, equation (2) can be used for computing the effective resistivity and equation (3) to estimate the relative fractional volume of rock and matrix. Figure 2 presents the ratio of the effective resistivity normalized by the matrix 
value $\left(\rho_{0} / \rho_{f}\right)$ as a function of the percentage of inclusions, for 2 different contrasts of resistivity between matrix and inclusions $\left(\rho_{i} / \rho_{f}=10\right.$ and 100) and for $m=2$. On the same graph is plotted the homogenisation curve of the bulk modulus (Christensen \& Lo, 1979) for a shear wave velocity contrast of 10. For the same contrast value, the electrical effective resistivity appears more sensitive to the presence of inclusions than the effective bulk modulus until an inclusion percentage of $80 \%$. Insofar as electrical properties of natural materials vary in a wider range than the seismic ones, the electrical tomography technique seems to be a promising method to investigate heterogeneous soils where resistive particles are present.

This paper proposes a methodology to estimate the composition of coarse heterogeneous soils using electrical measurements. Our approach articulates in three steps. The first step involves the use of finite element analysis (F.E.A.) to simulate electrical tomography experiments on a diphasic soil with known characteristics. The objective was to specify the required conditions for homogenizing the soil resistivity and to compare the numerical results with the predictions of the theoretical laws. In the second step, these requirements were tested using measurements performed on a small-scale laboratory model, where the concentration of resistive inclusions was known. In the last step, the methodology was applied to a real experimental site, where grain size analyses were performed on several samples in order to verify the predictions derived from electrical and electromagnetic measurements.

\section{NUMERICAL MODELLING OF ELECTRICAL TOMOGRAPHY}


Electrical Tomography is now a classical and widely used multi-electrode method for imaging the subsurface (see, for instance, Reynolds, 1997). In this study, numerical modelling is used to simulate the results of electrical tomography measurements in a heterogeneous medium. The finite-element code CESAR-LCPC (Mestat, 1997; Humbert, 1989) was used for simulating the current flow diffusion at steady state (DC measurements). As the maximum number of finite elements in the code was limited to $1.10^{5}$, most simulations were performed in two dimensions, with current line sources instead of point sources. This implies the reformulation of the geometrical factor $k$. In two dimensions and for a four electrode layout $(\mathrm{A}, \mathrm{B}, \mathrm{M}$ and $\mathrm{N})$, the resistivity is given by:

$$
\rho_{2 D}=k \frac{\Delta V_{2 D}}{I}=\frac{\pi}{\ln \left(\frac{M B \times N A}{M A \times N B}\right)} \frac{\Delta V_{2 D}}{I}
$$

where $I$ is the current injected between electrodes $\mathrm{A}$ and $\mathrm{B}$, and $\Delta V_{2 D}$ is the difference of potential between electrodes $\mathrm{M}$ and $\mathrm{N}$. For a Wenner alpha array (identical interspacing $a$ between the electrodes), equation (4) simplifies in:

$$
\rho_{2 D}=\frac{\pi}{2 \cdot \ln 2} \frac{\Delta V_{2 D}}{I}
$$

If the medium is heterogeneous, the measured resistivity values are apparent resistivities which have to be inverted in order to obtain an image of the subsurface with real resistivity values. This process can be achieved using, for example, the software RES2DINV (Loke \& Barker, 1996). In the following, we will use it to validate the homogenisation of the medium by comparing the apparent and real resistivity values. 


\section{Description of the model and preliminary tests}

The model structure (figure 3 ) is composed of a heterogeneous rectangular body (dimensions $W$ and $T$, resistivity $\rho_{f}$ ) containing circular resistive inclusions having all the same properties (radius $R$, resistivity $\rho_{i}$ ) and bordered by an external homogeneous zone (resistivity $\rho_{\mathrm{e}}$ ). Various numerical tests were conducted in order to ensure the validity of the F.E.A. model, using a mesh composed of three-node elements.

The inclusions were non-jointed and randomly spread. As the current paths are mainly contained in the conductive matrix, the presence of at least two layers of finite element was required between two neighbouring inclusions, in order to not limit numerically the conduction of these areas. A minimal distance of $0.2 R$ was needed between two inclusions to ensure a sufficient spatial discretization, limiting the possible percentage of inclusions to $40 \%$. Moreover a minimum of two three-node elements between two neighbouring electrodes was required. Such a dense spatial meshing limited the possible size $(W \times T)$ of the heterogeneous rectangular body, the resistivity $\rho_{o}$ of which were studied.

The external zone was defined in order to move the boundaries of the mesh (Dirichlet condition, $V=0$ ) away from the central investigated area. It was shown that the distance $L$ between the centre of the mesh and its boundaries (figure 3) must be 15 times greater than the electrode spacing $a$, to contain the error on resistivity values below $0.5 \%$. So the simulated sequence of measurements (with spacing varying from $a_{\min }$ to $a_{\max }$ ) had to respect the condition $L / a_{\max } \geq 15$.

The resistivity of the external zone $\rho_{\mathrm{e}}$ was fixed thanks to Bussian's relashionship, using a cementation factor $m=2$ (2D, circular inclusions). The influence of this area on the current paths was analysed and a correction procedure was set up to clear the results from this disturbance. 
Homogenising a heterogeneous soil requires an electrode spacing $a$ larger than the radius $(\mathrm{R})$ of inclusions. In order to define this condition, a sequence consisting of more than 1000 Wenner-alpha arrays was simulated on a mesh containing $40 \%$ volume of resistive inclusions $\left(\rho_{i}=100 \rho_{f}\right)$. The ratio $a / R$ was calculated for each single measurement (spacing $a$ ), and varies between 1 and 25 on the whole sequence. The numerical results (figure 4 ) proved that over a value of ratio $a / R$ equal to 8 , the average resistivity becomes stable and the scattering significantly decreases, indicating that the soil is seen to be homogeneous by the method. Similar results were obtained with a concentration of inclusions of $25 \%$.

\section{Results}

Once that the different geometrical requirements were defined $\left(a_{\min } / R=8 ; L / a_{\max }=15 ; \ldots\right)$ computations were performed for two different contrasts of resistivity $\rho_{i} / \rho_{f}(10$ and 100). A sequence of 135 measurements using Wenner alpha array was applied on each mesh representing a random distribution of inclusions, and the mean apparent resistivity was calculated on each pseudosection. Numerical results for 50 random concentrations varying in the range $0-40 \%$ are plotted on figure 5. They were compared to the theoretical laws (equation 3 ) with a cementation factor $m$ equal to 2 . Figure 5 shows a good fit between numerical results and theoretical laws, RMS values ranging from 3.6 to $4.0 \%$ for the four tested resistivity contrasts $\left(\rho_{i} / \rho_{f}=10,20,50\right.$ and 100). Only the two extremes are shown in figure 5. Similar good agreements were found modelling square inclusions with a different software (Rey et al., 2003). Twelve of the simulated pseudo-sections (whose average resistivity is presented) on figure 5 were inverted using the Res2DInv software (Loke \& Barker, 1996). No significant difference was observed between the mean apparent resistivity 
(pseudo-section) and the mean inverted resistivity. Consequently, effective resistivity can be directly derived by averaging apparent resistivity values.

This result confirms that the electrical tomography method homogenises correctly a heterogeneous soil when the condition specified here above $\left(a_{\min } / R\right.$ greater than 8$)$ is verified, and consequently that theoretical law can be used.

\section{SMALL-SCALE PHYSICAL MODEL}

\section{Description}

In order to compare the theoretical predictions to real data, we constructed a small-scale physical model consisting of a composite heterogeneous medium. A bin with dimensions $2 \times 1 \times 0.5 \mathrm{~m}$ was built out of marine plywood and filled with resistive wooden inclusions of one meter long, which were embedded in a matrix of silty sand (figures $6 a, 6 b)$. These rods, of square cross-section $(27 \times 27$ $\mathrm{mm}$ ), were spaced at $13 \mathrm{~mm}$ intervals and set up in 12 layers of $40 \mathrm{~mm}$, including the silty sand thickness (figure 6c). Inclusion volume concentration was uniformly distributed and equal to $44.3 \%$. The structure can be considered as two-dimensional. The silty sand was generated from crushing a sandy limestone. It was sieved at $5 \mathrm{~mm}$ and contains an important percentage of $80 \mu \mathrm{m}$ weight undersize (30 to $40 \%$ ) and a moderate clay fraction (VBS $=0.6$ at the methylene blue test, French Standard NF P 094-068). It was saturated to increase its conductivity and the water content was monitored.

\section{Measurements}

A reduced-scale measurement device was developed, consisting of 16 electrodes made of brass, with a diameter of $4 \mathrm{~mm}$ and a length of $150 \mathrm{~mm}$. A wooden support was limiting their sinkage to 10 
$\mathrm{mm}$. They were spaced every $110 \mathrm{~mm}$ (figure $6 \mathrm{~d}$ ), so the homogenization condition $\left(a_{\min } / R \geq 8\right.$ ) was respected since the inclusions are assumed to be cylindrical rods with a radius of $13.5 \mathrm{~mm}$. A sequence of measurements was carried out on the full bin with an Iris Syscal R1+ as resistivity recorder.

The raw pseudo-section (figure 7a) shows an important vertical gradient of resistivity due to the effect of the marine plywood envelop box whose resistivity is about 1000 times greater than its content. The 3D-effect of the resistive box was simulated with the finite-element code and the corresponding correction was applied on the raw data. The corrected pseudo-section (figure 7b) shows a slight negative gradient when depth increases, which remained after inversion (figure 7c). On the investigated part of the model (0-25 $\mathrm{cm}$ depth), the average (effective) resistivity value presents a negligible variation during the inversion process (figure 8), varying from 115 +/- 19 S.m for the raw data to $114+/-34 \Omega . m$ after 6 iterations (uncertainty is given by the standard deviation computed on the tomography section). The major effect of inversion process was an increase of the scattering of the resistivity values.

\section{Resistivity of each component}

In parallel the resistivities of each component were measured in order to interpret the resistivity of the model in terms of concentration of inclusions. The bin was emptied by removing 3 layers at a time. At each stage, one sample of silty sand and 5 samples of wood were taken. DC resistivity was measured on each sample using a HP-34410 impedance meter with an input impedance of $10 \mathrm{G} \Omega$. As the wood samples were cube-shaped, the resistivity measurements were performed in the three directions and the mean value (574 $\Omega . \mathrm{m})$ was kept. Silty sand samples were enclosed in a nonconductive PVC cylindrical holder with an inner diameter of $21 \mathrm{~mm}$ and a length of $50 \mathrm{~mm}$ and 
closed by two metallic electrode plates. Figure 9 shows the resistivity of the silty sand, which decreases with depth (from 45-50 in the upper part to $15 \Omega . \mathrm{m}$ at the bottom of the bin), as for the whole model. This vertical gradient of resistivity can not result from variations of the water content because the water alone was more resistive (between 20 and $23 \Omega . m$ ) than the saturated silty sand (15 $\Omega . \mathrm{m}$ at the bottom of the bin). Such a low resistivity can be explained by the well-known surface conductance phenomena, which can lead a porous material to be more conductive than its pore fluid (for example, Revil et al., 2002). The vertical gradient of resistivity for the silty sand can be a consequence of a higher percentage of fine particles on the bottom than on the top of the model (38.5\% of $80 \mu \mathrm{m}$ weight undersize measured on the bottom of the bin against $31.7 \%$ on the top). Fine particles must have been dragged towards the bottom by the water percolation, during the saturation process. On the investigated part of the model $(0-25 \mathrm{~cm}$ depth), the resistivity of the silty sand is nearly stable and the average value (48 $\Omega . \mathrm{m})$ was considered to estimate the model composition.

\section{Interpretation and discussion}

Since numerical computations validated the use of Bussian's theoretical law to predict effective resistivity when the spacing $a_{\min }$ of the smallest measurements is greater than or equal to $8 R$, equation (3) can be used to estimate the concentration of resistive inclusions in the physical model. So, considering the average resistivity of the mixture (115 $\Omega . \mathrm{m})$, the resistivity of the wood $(574 \Omega . \mathrm{m})$ and of the silty sand (48 $\Omega . \mathrm{m}$ ), the fractional volume of the resistive inclusions present in the model was estimated to $43.7 \%$, using $m=2$ (real $44.3 \%$ ). The chosen value for the cementation factor is supported by the $2 \mathrm{D}$ structure within the bin. Indeed the infinitely long rods (compared to their equivalent radius) can be considered at first approximation as everywhere perpendicular to the 
electrical field. The exact concentration $(44.3 \%)$ is reached for a $m$ value of 1.95 . This slight decrease can be explained by the 3D current propagation ( $m=1.5$ in 3D). Nevertheless these results prove, as for numerical modelling, that Bussian's relationships can be used to link the effective resistivity of a mixture to its characteristics with a good precision.

\section{FIELD MEASUREMENTS}

\section{Experimental site}

To apply our methodology under real conditions, an experimental site was investigated, localised 15 km North of Grenoble, in the Gresivaudan Valley (France). This important NE-SW glacial groove separates the crystalline mountain chain of Belledonne to the East and the sedimentary mountain chain of Chartreuse located to the West. On the eastern boundary of the latter range of mountain, the sedimentary formations are eroded perpendicularly to the Gresivaudan Valley (toward NW) by the ephemeral stream torrent Manival (see figure 10). Its alluvial-fan deposits results from the erosion of two geological formations: Argovian marlstone and Sequanian marly limestones. Rocks are mainly produced by the fracturing of the marly limestone whereas clays and fines originate from the alteration of marlstone.

Three geophysical profiles were defined in the upper part of the alluvial-fan, consisting of an electrical tomography and inductive electromagnetic measurements (Geonics EM31-D). Advantages of the latter method are its rapidity and a direct spatial integration of the apparent resistivity on the upper most 3-6 superficial meters. After these measurements, the ground was excavated vertically at each profile location and sampled at different points in order to compare the real grain size distributions with the estimated block concentration deduced from geophysics. 


\section{Geotechnical data}

Dimension of the largest particles $(0.8 \mathrm{~m})$ observed on the field surface would require samples weighing more than 250 tons, according to the French Standard (NFP 94-056). For obvious practical reasons samples of 2 to 3 tons only were taken. After successive quartering and in-situ sieving, a mass of $20 \mathrm{~kg}$ of fraction $0 / 50 \mathrm{~mm}$ for each sample was brought back to the laboratory to undergo a complete grain size analysis and water content measurements. Each distribution shows the presence of a plateau for fine particle sizes and a clear change in gradient of each curve is observed from 5 to $10 \mathrm{~mm}$ (figure 11). So, the soil contains both fine particles and a significant proportion of rock pieces with diameter larger than $10 \mathrm{~mm}$.

\section{Geoelectrical measurements}

\section{Field experiments}

Each tomography profile was composed of 32 electrodes with a minimal spacing of $0.8 \mathrm{~m}$ (diameter of the largest particles) in order to investigate the upper most four meters, depth of investigation being about one sixth of the profile length. Tomographies were performed using both Wenner-alpha and dipole-dipole arrays. Mean resistivity was calculated before and after inversion (table 1) keeping for each profile only representative spacing values $(a \geq 8 R)$. The inversion process generates a slight increase of the average values, compared to the apparent resistivity values.

Electromagnetic measurements were performed along the same profiles, every one meter, with an intercoil spacing of 3.66 meters parallel to the profiles. The used frequency $(9.8 \mathrm{kHz})$ and the observed range of resistivity allow to investigate the upper most 6 meters.

At the scale of the whole site, apparent measured resistivities are the same whatever the used technique (table 1). At the scale of each profile, electromagnetic measurements appears less sensitive 
to the lateral slight variations of resistivity, as shown on figure 12 representing in parallel EM and tomographic data for the P2 profile. It is probably due to its deeper integration, as well as the fact that electrical tomography seems more open to lateral zoning.

\section{Determination of the matrix and inclusion resistivity values}

In parallel, electrical characteristics of each component have been measured at two different scales (Table 2). At the scale of the geological formations we installed short profiles (32 electrodes with spacing of $0.5 \mathrm{~m}$ ) separately on outcrops of each formation located near the top of the catchment area. At a smaller scale, measurements on limestone were performed in the laboratory on cores using the same impedance meter as described therein. A corresponding value for fine particles was obtained using the field equipment with four-electrode spaced of a few tens of centimetres and driven in flood sediments. These deposits contained only particles of diameter less than $1 \mathrm{~mm}$. Since measurements showed similar results whatever the scale, the following values can be kept: $\rho_{i}=690$ +/- $70 \Omega$.m for limestone blocks and $\rho_{f}=50+/-3 \Omega$.m for matrix resistivity, considering that the resistivity of the marlstone is more a maximum boundary for the fine particles resistivity than an exact value. Then the contrast of resistivity $\rho_{i} / \rho_{f}$ between inclusions and matrix is equal to $14+/-2$.

\section{Interpretation and discussion}

On a large scale (i.e., the entire site), the inversion process does not modify significantly the mean resistivity value (less than $7 \%$ ), so the average raw resistivity (181 $\Omega . \mathrm{m})$ was used for interpretation. After normalization by the matrix value, the obtained ratio $\rho_{0} / \rho_{f}$ of 3.63 leads to estimate the concentration of resistive heterogeneities to $66+/-5 \%$ (using $m=1.5$ as seen above for dispersed spheres). The corresponding percentage volume of fine particles $(34+/-5 \%)$, when reported on the 
grain size distributions of the samples allows us to estimate the limit of size between what is considered as rocks or matrix. This mean size of $12+/-4 \mathrm{~mm}$ is in a good agreement with the change in gradient on curves observed on the undersize distributions (figure 11).

For such material the grain size distribution is particularly interesting to determine and the first step consisting in the identification of the change in gradient on curves was successfull.

\section{CONCLUSIONS}

Our study was dedicated to estimate the potential of a classical geophysical method to characterize in a geotechnical sense the heterogeneous soils containing particles of decimetre to metre size. Among different geophysical methods, electrical tomography, which is now widely used for shallow investigation, was tested for estimating the concentration of resistive inclusions in a soil with a conductive matrix. Three approaches have been investigated: finite element modelling and comparison with existing theoretical homogeneization laws, small scale laboratory experiments and field measurements on a real site presenting limestone blocks embedded in a marly matrix. Finite element modelling and small scale laboratory experiments allowed us to define the conditions necessary to obtain equivalent homogeneous (effective) resistivity of heterogeneous materials. Numerical modelling showed that resistivity measurements homogenize correctly the heterogeneous soil for electrode spacing (Wenner array) 8 times larger than the inclusion radius. Also, numerical results validated the theoretical homogenization law of Bussian (1983) for the scale of decimetre to meter size inclusions. A critical parameter of the Bussian law is the cementation factor which depends of the particle shape and the current flow pattern (2D or 3D). For circular inclusions in a 2D model, $\mathrm{m}$ is equal to 2 and decrease to $1.5 \mathrm{in}$ a $3 \mathrm{D}$ model for spherical inclusions. Using this homogenization law, resistivity measurements are able to provide an estimation of the inclusion percentage if the 
resistivity of the two phases is known. This method was satisfyingly confronted to a laboratory experiment, for which the inclusion concentration was known. Finally, mean resistivities were deduced from electrical tomographies and inductive EM measurements on a real site, where the grain size distribution was independently characterized. Our measurements and interpretation showed that the obtained limestone particle concentrations were consistent with the grain size data. Compared to surface wave acquisition and processing, which also provide an estimation of the particle concentration in the ground (Chammas et al. 2003), the main advantages of resistivity measurements are their rapidity and the ability of obtaining a 2D image allowing lateral variations of particle concentrations to be detected on a site.

\section{ACKNOWLEDGEMENTS}

The authors thank André Revil (CEREGE Aix-en-Provence, France) for allowing us to use his equipment and for interesting and helpful discussions. We are grateful to Philippe Côte and Rabi Chammas (LCPC Nantes, France) for their cooperation and informed advices about the finite element code CESAR. LIRIGM is a member of the French network RNVO. This research was supported by the "Pôle Grenoblois des Risques Naturels".

\section{REFERENCES}


Abraham O., Chammas R., Côte Ph., Pedersen H.A., Semblat J.F., 2004. Mechanical Characterisation of heterogeneous soils with surface waves : experimental validation on reducedscale physical models. Near Surface Geophysics, Vol. 2, n²4, 247- 258.

Archie G. E., 1942. The electrical resistivity $\log$ as an aid in determining some reservoir characteristics. Petr. Tech. 1, 55-62.

Bourdeau Y., 1997. Le comportement des alluvions du Rhône dans une grande boîte de cisaillement direct. Revue Française de Géotechnique 79, 45-57.

Bussian A.E., 1983. Electrical conductance in a porous medium. Geophysics, v.48, pp 1258-1268.

Chammas R., Abraham O., Côte Ph., Pedersen H.A., Semblat J.F., 2003. Characterization of heterogeneous soils using surface waves : homogenization and numerical modelling. International Journal of Geomechanics 3, 55-63.

Christensen R.M., Lo K.H., 1979. Solution for effective shear properties in three phase sphere and cylinder models. J. Mech. Phys. Solids 27, 315-330.

Fagnoul A. et Bonnechere F., 1969. Shear strengh of porphyry materials. Proceedings of the $7^{\text {th }}$ International Conference on Soil Mechanics and Foundation Engineering. Special session Mexico, 23-28 august 1969, 13, E1, 61-65. 
French Standard NF P 94-056. Sols : Reconnaissance et essais. Analyse granulométrique. Méthode par tamisage à sec après lavage. Ed. AFNOR, T1, 15p., 1996.

French Standard NFP 94-068. Sols : Reconnaissance et essais. Mesure de la quantité d'adsorption de bleu de méthylène d'un sol ou d'un matériau rocheux. Détermination de la valeur au bleu de méthylène d'un sol ou d'un matériau rocheux par l'essai à la tache. Ed. AFNOR, T1, 7p., 1998.

Holtz W.G., 1961. Triaxial shear characteristics of clayey gravel soils. Proceedings of the $5^{\text {th }}$ International Conference on Soil Mechanics and Foundation Engineering, Paris, 1961, 143149

Humbert P., 1989. CESAR-LCPC : Un code général de calcul par éléments finis. Bull. liaison des Laboratoires des Ponts et Chaussées 160, 112-115

Jackson P. D., Taylor Smith D., Stanford P. N., 1978. Resitivity-porosity-particle shape relationships for marine sands. Geophysics 43, 1250-1268.

Jongmans D. , Demanet D., 1993. The importance of surface waves in vibration study and the use of Rayleigh waves for estimating the dynamics characteristics of soils. Engineering geology 34, 105113.

Loke M.H. \& Barker R.D., 1996. Rapid least-squares inversion of apparent resistivity pseudosections by a quasi-Newton method, Geophysical Prospecting 44, 131-152. 
Mayne P. W., Rix G. J., 1995. Correlations between shear wave velocity and cone tip resistance in natural clays . Soil and Foundations $35, \mathrm{n}^{\circ} 2,107-110$

Mendelson K. S., Cohen M.H., 1982. The effect of grain anisotropy on the electrical properties of sedimentary rocks to the dielectric constant of fused glass beads. Geophysics 47, 257-263.

Mestat P., 1997. Maillages d'éléments finis pour les ouvrages de géotechnique. Conseils et recommandations. Bull.liaison des Laboratoires des Ponts et Chaussées 212, 39-64

Revil A., Hermitte D., Spangenberg E., Cochemé J.J., 2002. Electrical properties of zeolitized volcanoclastic materials. J. Geophys. Res. 107 (B8), doi:10.1029/2001JB000599

Rey E., Garambois S., Jongmans D., Gotteland P., 2003. Characterisation of coarse materials using geophysical methods. Proceedings of the $9^{\text {th }}$ Meeting of Environmental and Engineering Geophysics, Prague, O-061.

Reynolds J., 1997. An introduction to applied and environmental geophysics. Ed. Wiley, John \& Sons, Inc. 749 p.

Sen P.N., Scala C., Cohen M.H., 1981. A self-similar model for sedimentary rocks with application to the dielectric constant of fused glass beads. Geophysics 46, 781-795. 
Stokoe K.H., Nazarian S., Rix G.J., Sanchez-Salinero. I., Sheu J-C. and Mok Y.J., 1988. In situ seismic testing of hard-to-sample soils by surface wave method. Earthquake Engineering and Soil Dynamics II - Recent Advances in Ground Motion evaluation, Geotechnical special publication, 20, J.L. Von Thun, Ed. ASCE, New York, 64-278 


\section{FIGURE CAPTIONS}

Figure 1: (a) Photography of an heterogeneous soil made of limestone rocks and particles embedded in marly clay (Torrent Manival, France) - (b) Schematic view of a diphasic medium with resistive inclusions in a conductive matrix.

Figure 2: A: Normalised effective resistivity $\left(?_{d} ?_{f}\right)$ as a function of concentration of inclusions for a cementation factor $m=2$ and a resistivity contrast $?_{f} / ?_{i}=10$ (after Bussian, 1983). B: The same as A for a resistivity contrast of 100 . C: Normalised bulk modulus $K_{d} / K_{f}$ for a shear wave velocity contrast $\left(\mathrm{Vs}_{\mathrm{i}} / \mathrm{Vs}_{\mathrm{f}}\right)$ of 10 (Christensen and Lo, 1979).

Figure 3: Finite element model meshing used in the numerical modelling. $\mathrm{L}$ and $\mathrm{Z}$ denote respectively the half length and the depth of the entire model. The heterogeneous rectangular body (large white square contour, dimensions $W x T$ ) is located on the top at the centre of the meshing. The zoom shows that the host conductive medium (resistivity: ?f) is more densely meshed than the core of inclusions $\left(?_{\mathrm{i}}\right) . ?_{\mathrm{e}}$ is the resistivity of the external homogenous zone.

Figure 4: Normalized effective resistivity (numerical results) as a function of ratio $a / R$ for a concentration of heterogeneities of $40 \%$. The dashed line represents the relative variation of $?_{0} / ?_{\mathrm{f}}$, error band are statistical (standard deviation). Vertical lines delimit the range of $a / R$ for which the soil is seen to be homogeneous by the method (for $a / R>20$, the influence of the external homogeneous zone $\rho_{e}$ becomes predominant). 
Figure 5: Variation of the normalised effective resistivity as a function of the percentage of inclusions for two resistivity contrasts (10 and 100). Numerical results (apparent normalised resistivity) are computed for 50 random distributions of inclusions (with concentrations varying from 0 to $40 \%$ ). Theoretical curves are calculated using Bussian's law with a cementation factor $m=2$.

Figure 6: Small-scale laboratory model. (a) Global view of the bin. (b) Layout of the $1 \mathrm{~m}$ long wood rods embedded in silty sand. (c) Partial cross section showing the mixing of square rods and soil. (d) Small-scale electrical measurement device. If not precised, dimensions are in millimetres.

Figure 7: Results of laboratory electrical tomography measurements. Investigation of layer 0-25 cm depth : (a) pseudo-section data. (b) corrected pseudo-section data. (c) inverted resistivity model (here $\mathrm{RMS}=1.6 \%$ after 2 iterations).

Figure 8: Effective resistivity of the 25 most superficial centimetres of the model as a function of number of iterations. Full line represents the mean resistivity with associate standard deviation, and dashed line the RMS.

Figure 9: Laboratory resistivity measurements on silty sand as a function of the bin depth.

Figure 10: (a) Location of the real experimental site. (b) Global view of the erosion silted stream Manival. The investigated area is located by the white square contour (photo M. Gidon, www.geolalp.com). 
Figure 11: Grain size distributions (grey curves) of the seven samples taken in the Manival site. The thick dashed curve is the mean. The mean inclusion percentage $(66 \%)$ estimated by electrical measurements, corresponding to $34+/-5 \%$ undersize, leads to a size limit of about 12 (mean : bold arrow) $+/-4 \mathrm{~mm}$ (thin arrows) between matrix and inclusions.

Figure 12: Electrical measurements on profile P2. (a) Apparent resistivity data obtained from EM-31 measurements. (b) Measured pseudo-section from electrical tomography. Superficial non representative values have been removed. (c) calculated pseudo-section (Res2Dinv). (d) True resistivity model after inversion $(\mathrm{RMS}=2.9 \%)$. 

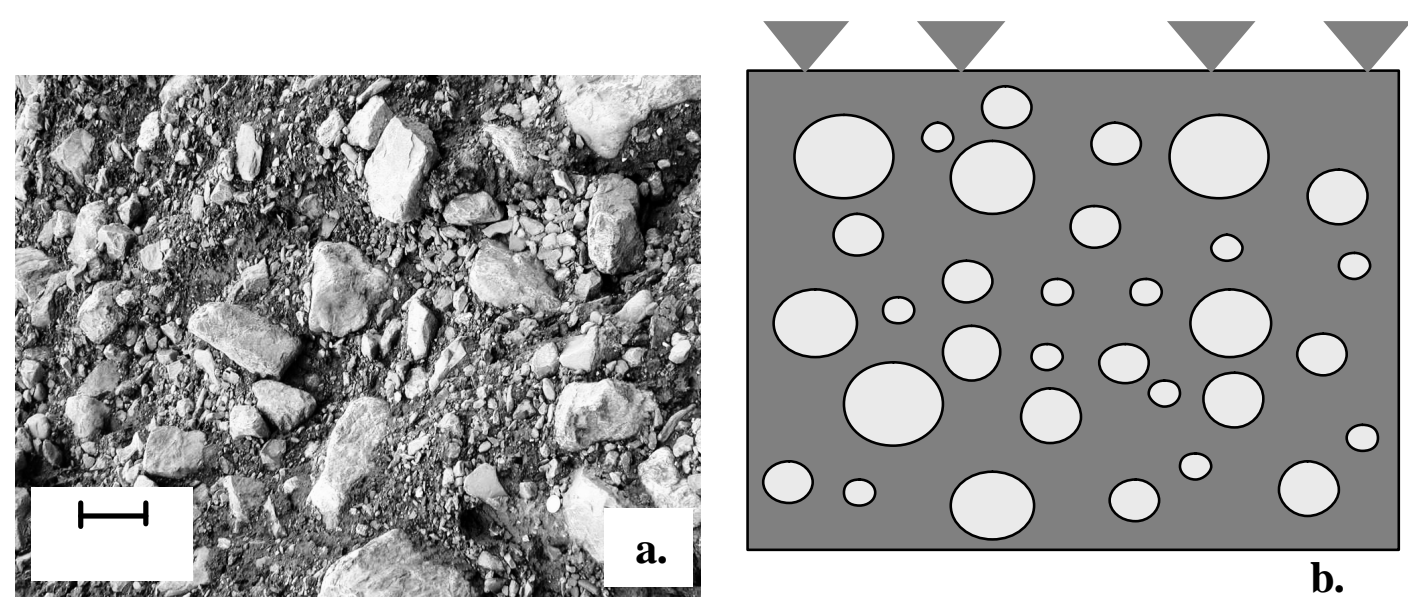

b.

Figure 1 


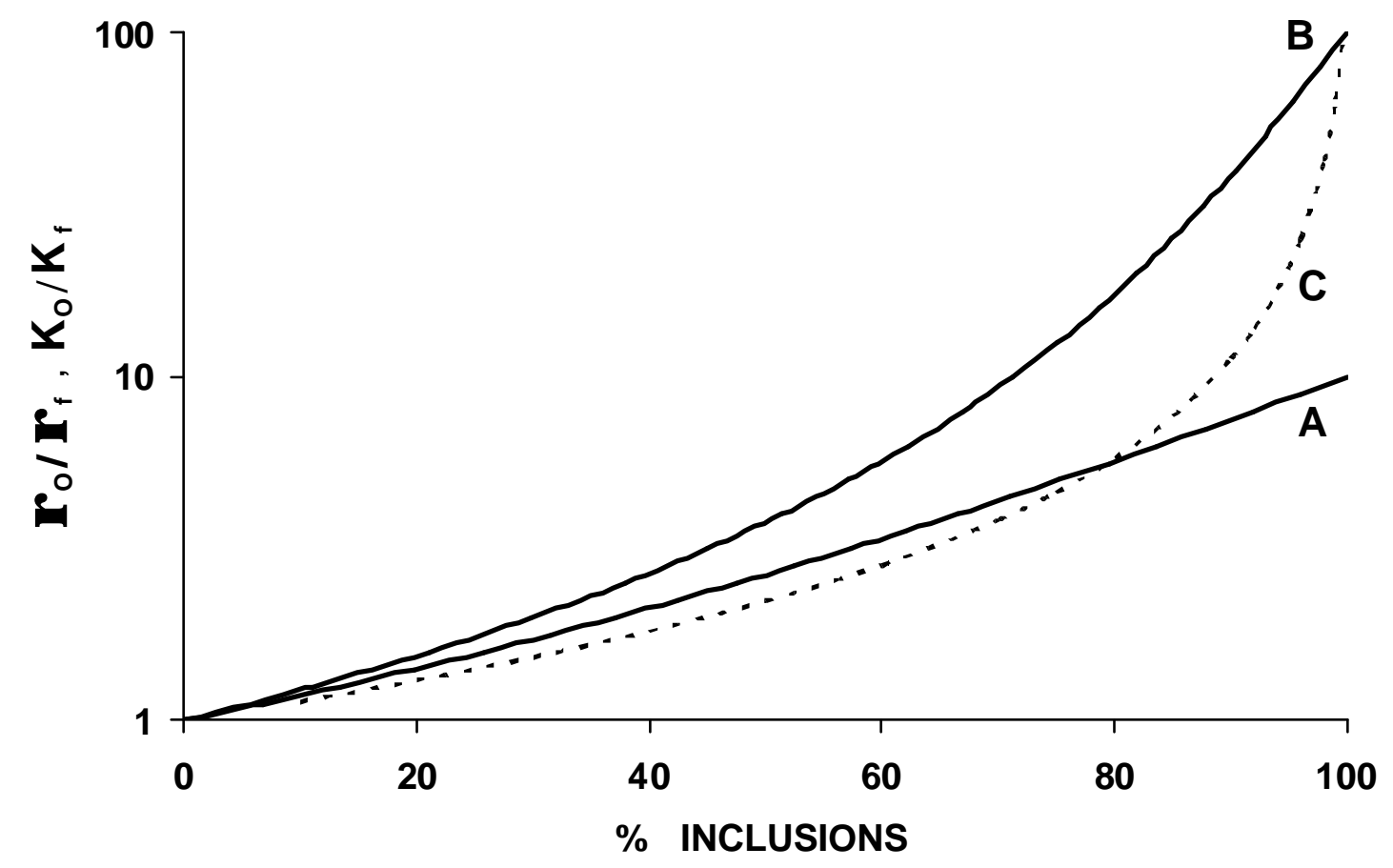

Figure 2 


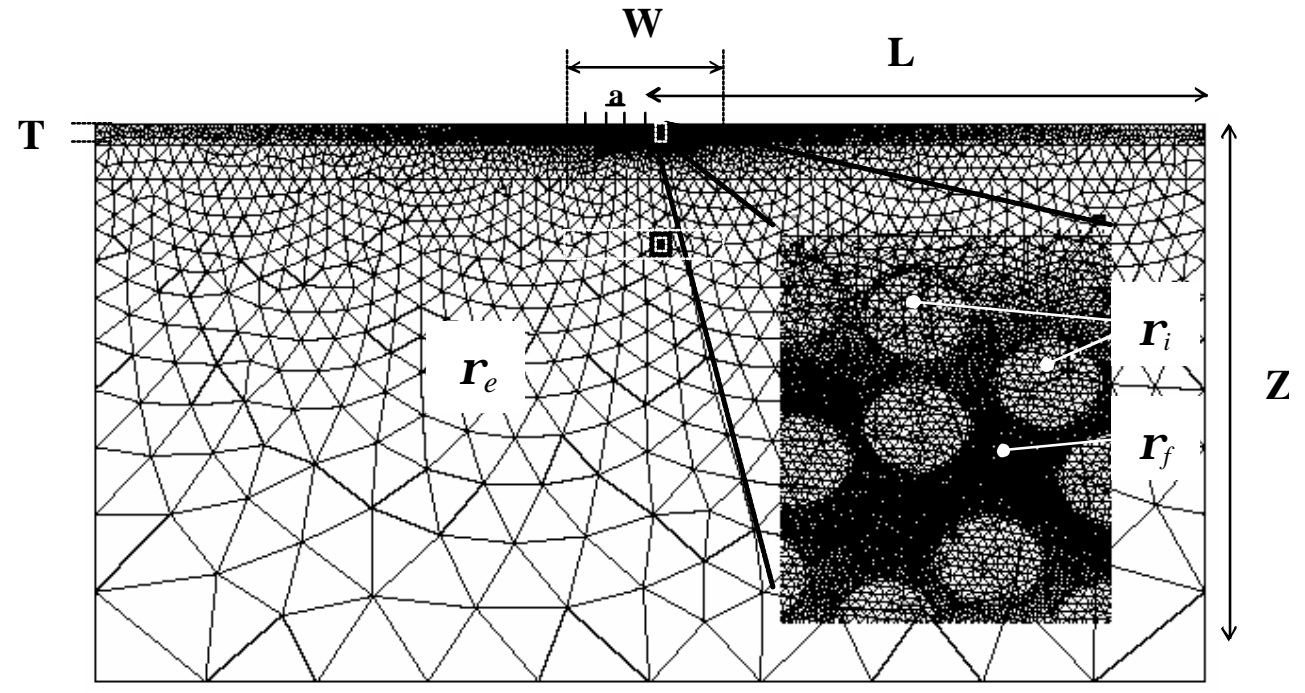

Figure 3 


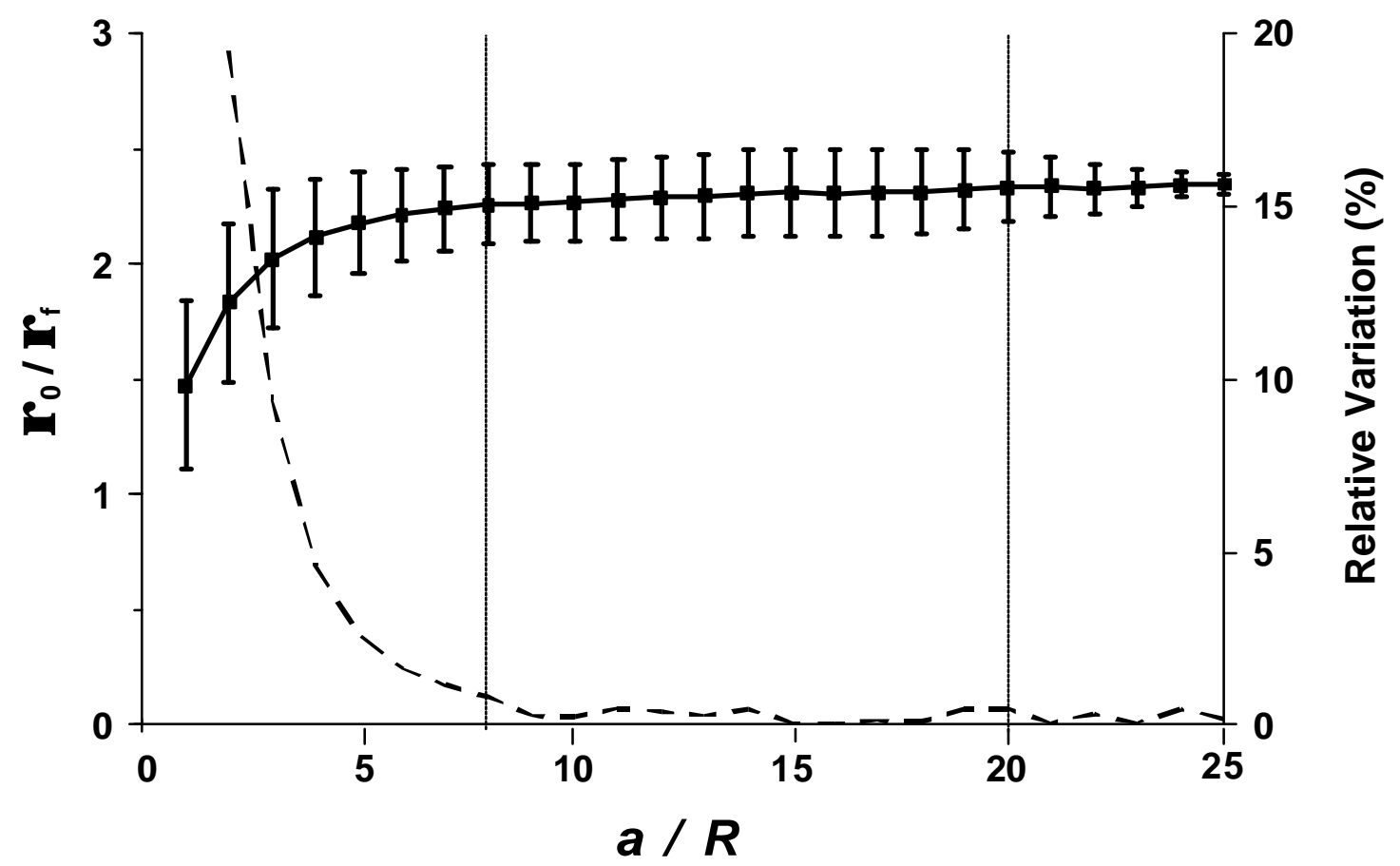

Figure 4 


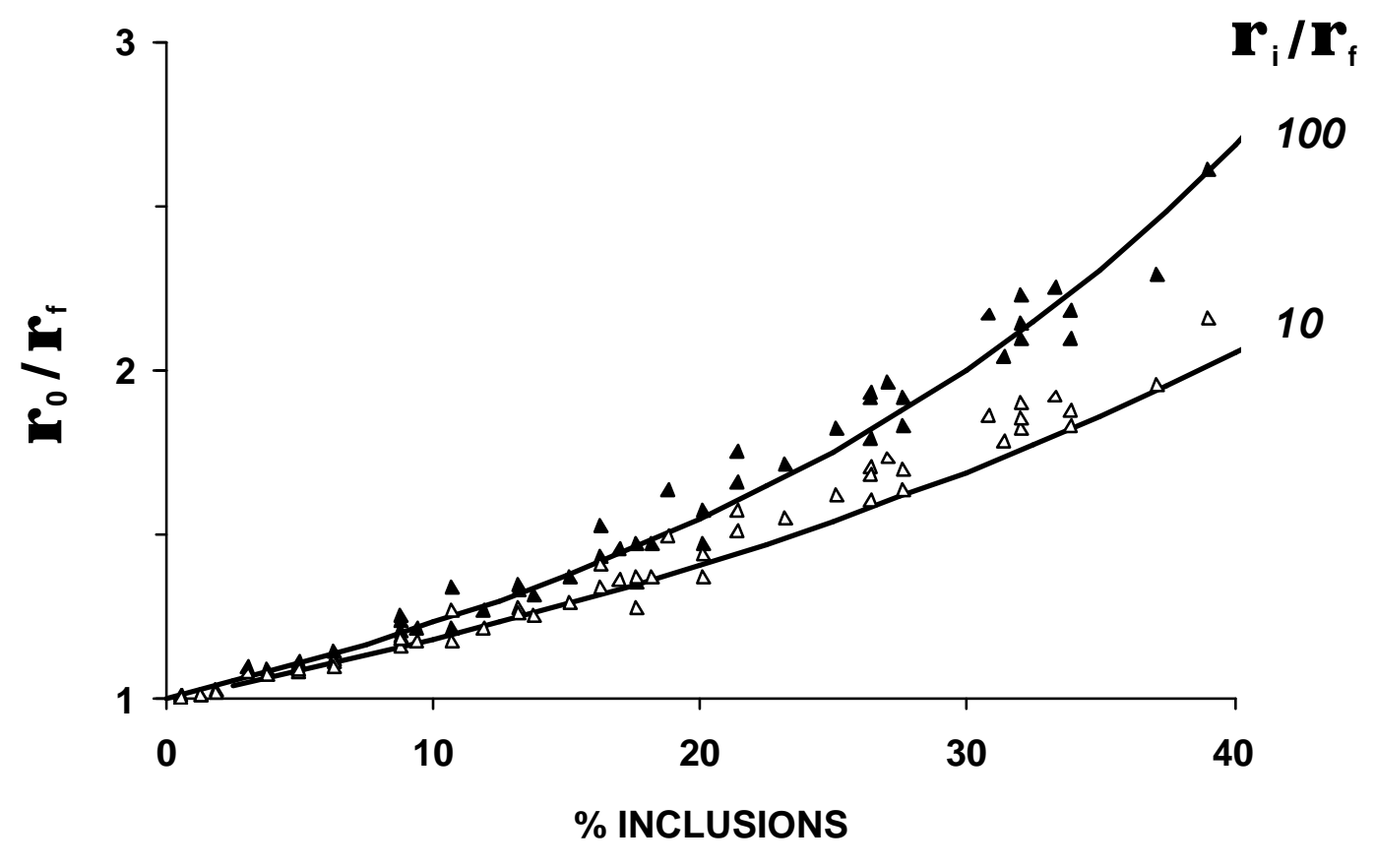

Figure 5 

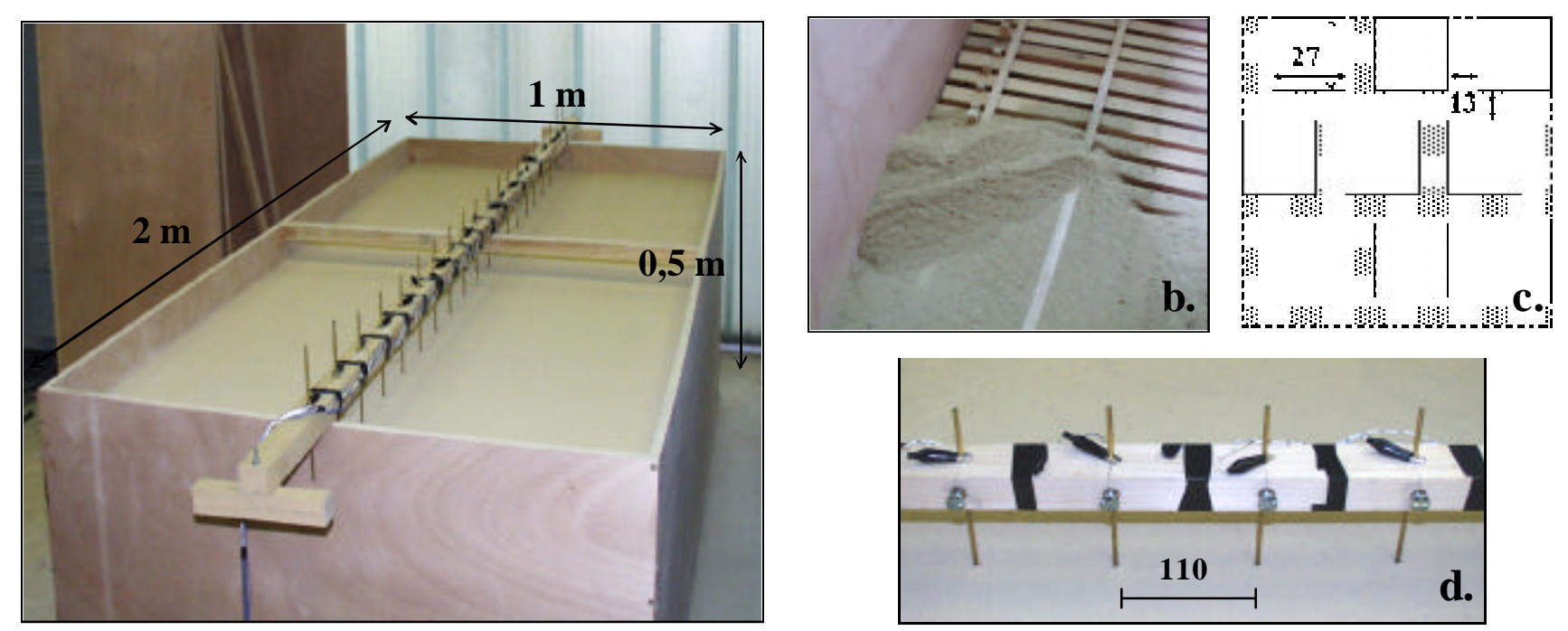

a.

Figure 6 


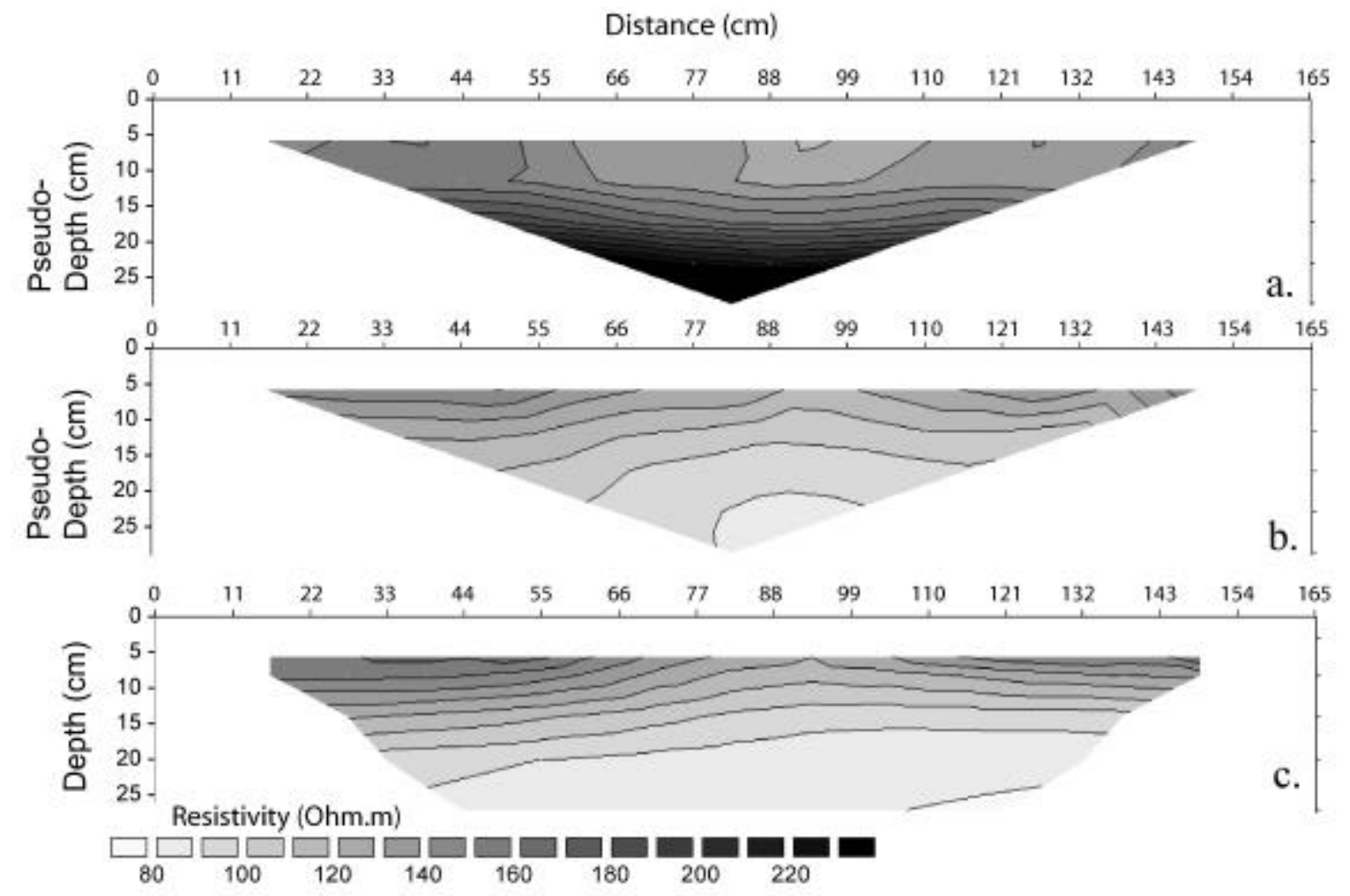

Figure 7 


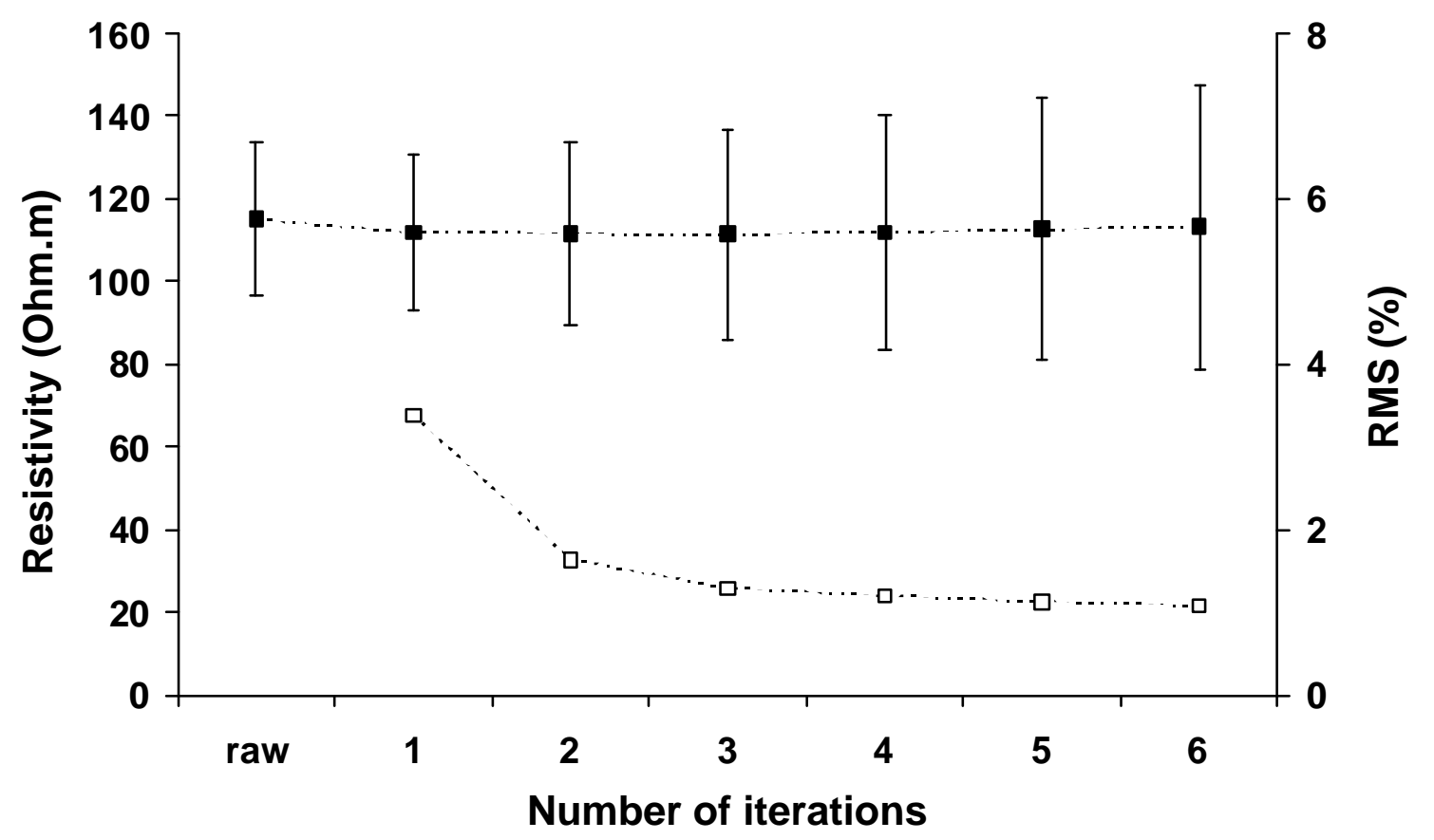

Figure 8 


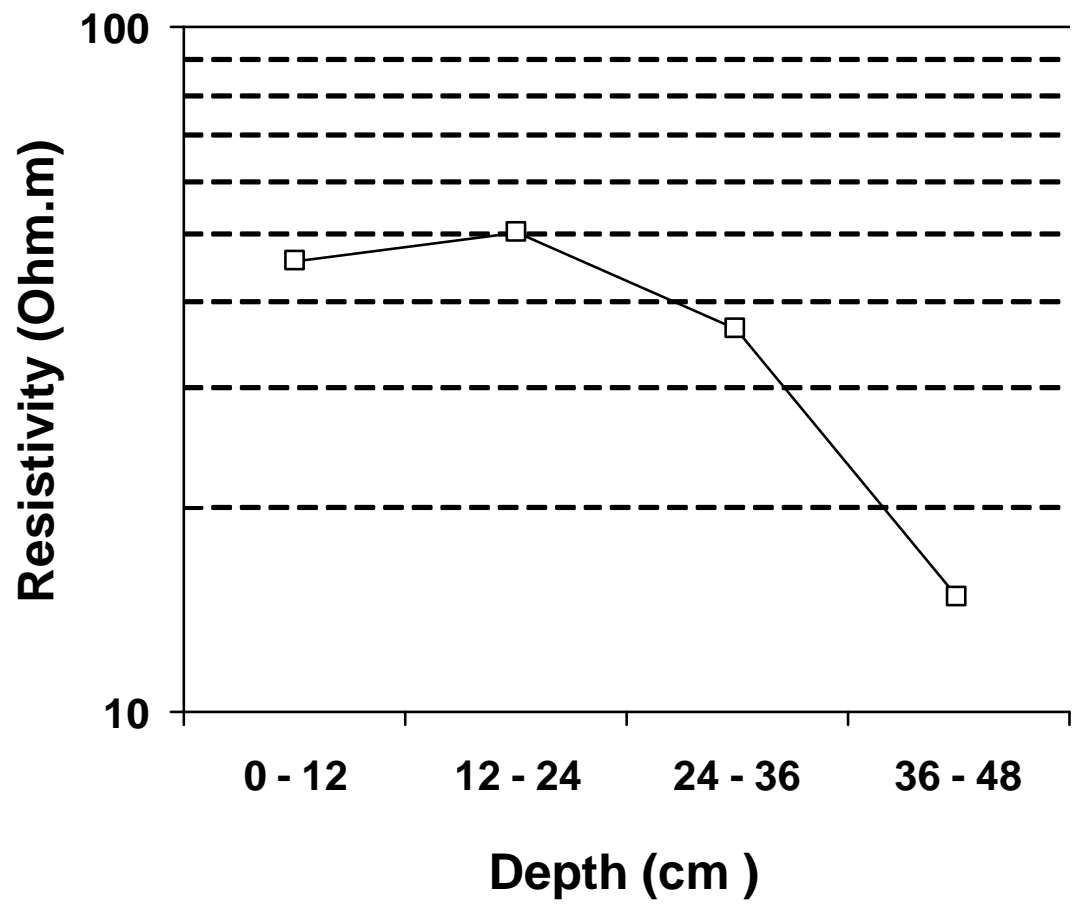

Figure 9 

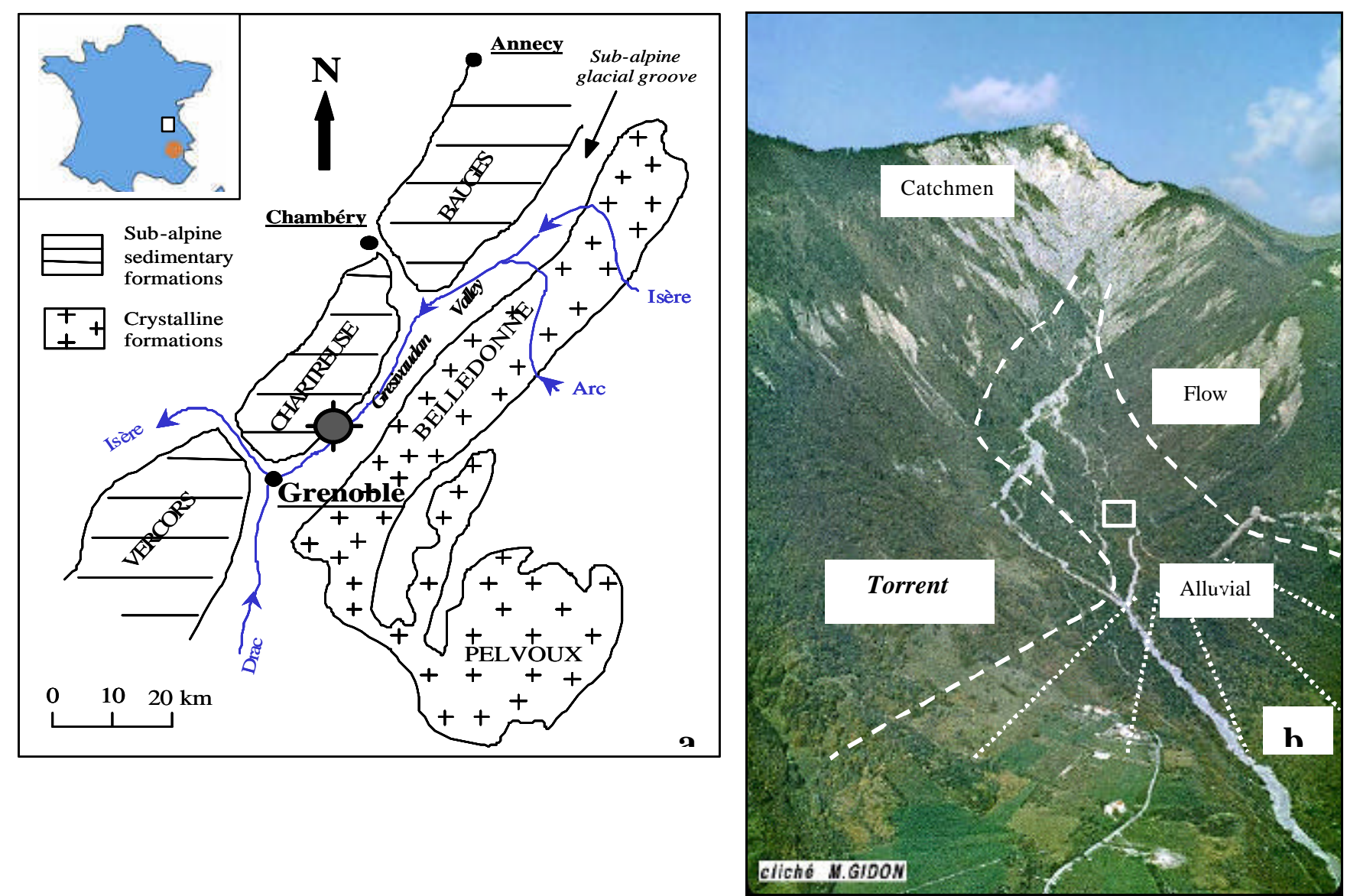

Figure 10 

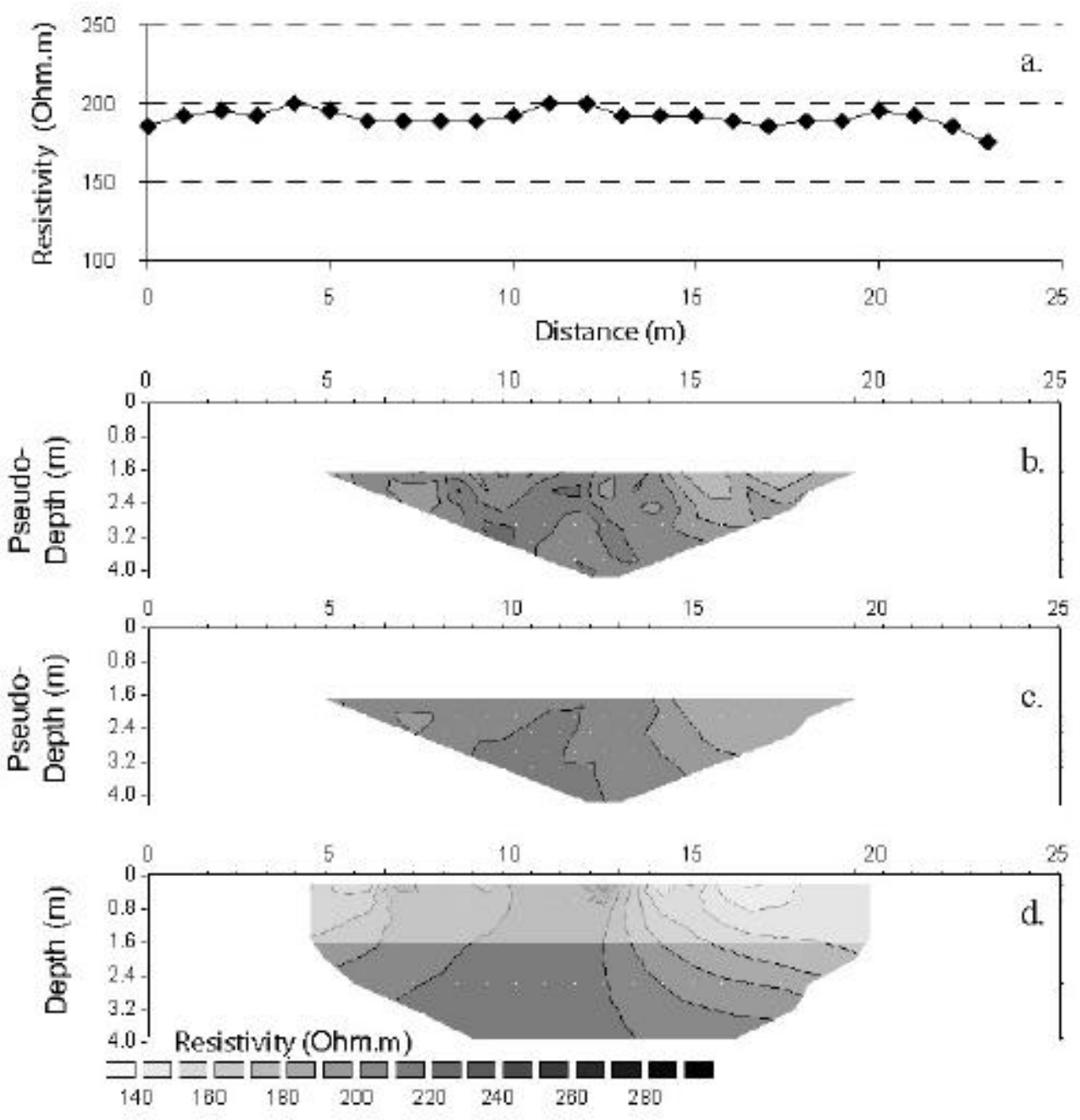

Figure 11 


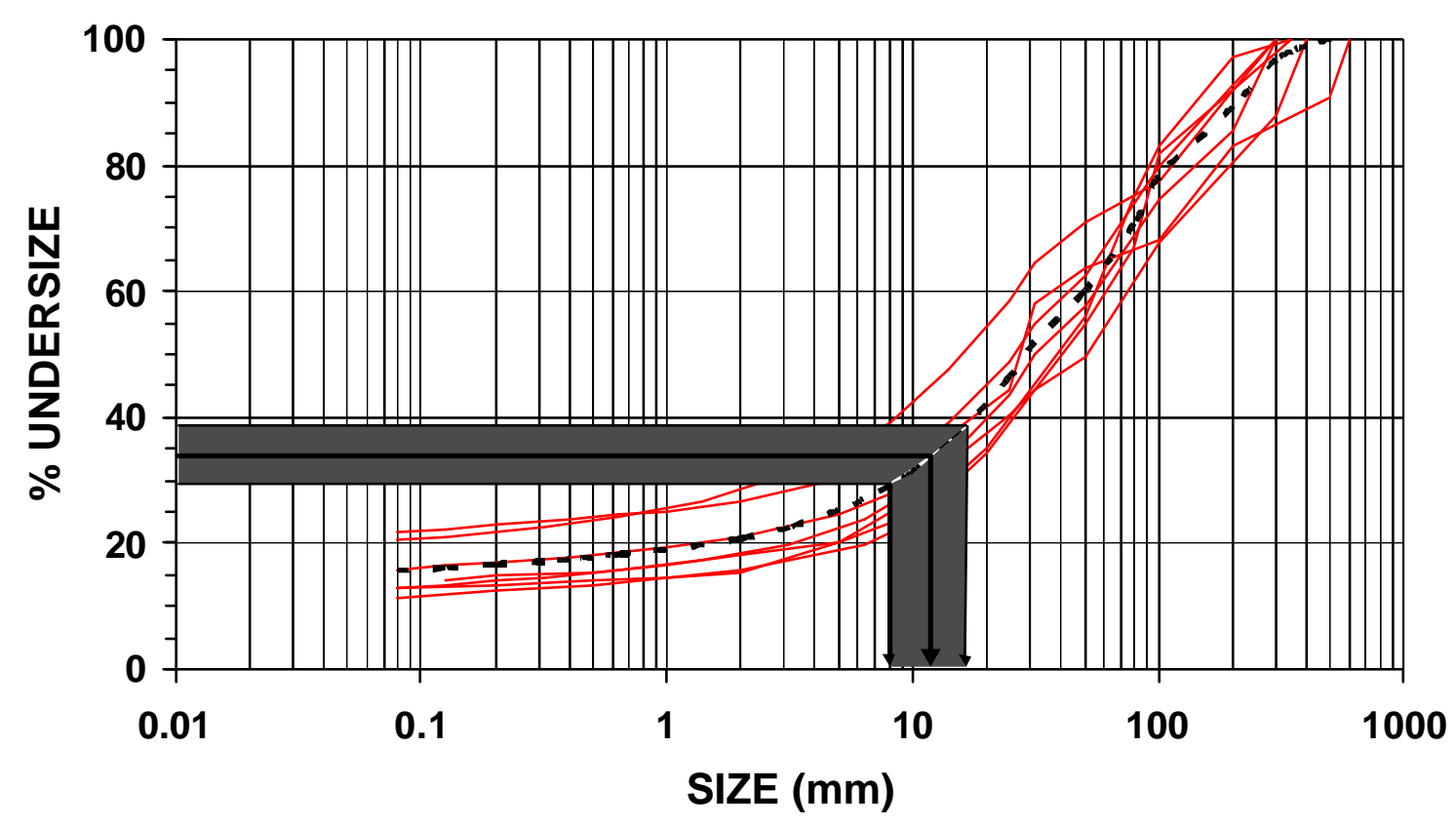

Figure 12 


\begin{tabular}{ccccc} 
Resistivity & Profile 1 & Profile 2 & Profile 3 & Site \\
\hline Apparent (DC) & $192+/-12$ & $201+/-13$ & $151+/-14$ & $181+/-26$ \\
\hline True (inverted) & $204+/-7$ & $209+/-13$ & $169+/-9$ & $193+/-20$ \\
$\%$ RMS & $2.5 \%$ & $2.9 \%$ & $3.4 \%$ & \\
\hline Apparent (EM 31) & $186+/-7$ & $191+/-5$ & $172+/-14$ & $183+/-12$ \\
\hline
\end{tabular}

Table 1 
Scale

$\rho_{\mathbf{i}}(\Omega . m)$

$\rho_{\mathrm{f}}(\Omega . \mathrm{m})$

Centimetric

Marly limestone :

665-880

Fines :

$50+/-3$

Decametric

Sequanian marly limestone : $690+/-70$

Argovian marlstone : 55+/- 3

Table 2 